\title{
Evidence-Based PET for Breast Cancer
}

\author{
Giorgio Treglia
}

\subsection{Introduction}

Fluorine-18 fluorodeoxyglucose positron emission tomography/computed tomography $\left({ }^{18} \mathrm{~F}\right.$ FDG PET/CT) is currently used in daily clinical practice for the evaluation of breast cancer (BC) patients. This chapter provides an overview of the current evidence-based data on the usefulness of PET/CT (using ${ }^{18} \mathrm{~F}-\mathrm{FDG}$ and other radiotracers) for different indications in patients with BC.

\subsection{Staging}

A recent network meta-analysis comparing 19 different imaging methods demonstrated the relatively higher specificity of ${ }^{18} \mathrm{~F}-\mathrm{FDG}$ PET/CT compared to other imaging methods for the detection of BC lesions [1].

\footnotetext{
G. Treglia $(\bowtie)$

Clinic of Nuclear Medicine and Molecular Imaging, Imaging Institute of Southern Switzerland, Ente Ospedaliero Cantonale,

Bellinzona and Lugano, Switzerland

Department of Nuclear Medicine and Molecular Imaging, Lausanne University Hospital and University of Lausanne, Lausanne, Switzerland

Health Technology Assessment Unit, Academic Education, Research and Innovation Area, Ente Ospedaliero Cantonale, Bellinzona, Switzerland e-mail: giorgio.treglia@eoc.ch
}

Liang et al. [2] evaluated through a metaanalytic approach the accuracy of magnetic resonance imaging (MRI) and ${ }^{18} \mathrm{~F}-\mathrm{FDG}$ PET/CT for lymph nodal (N) staging of early BC. The pooled specificities of MRI and PET/CT for diagnosing regional lymph nodal status in $\mathrm{BC}$ patients were similar (93\%); however, the pooled sensitivity of MRI was significantly greater than PET/CT (82\% versus 64\%), respectively.

Hong et al. [3] performed a meta-analysis to evaluate the value of ${ }^{18} \mathrm{~F}-\mathrm{FDG}$ PET/CT for diagnosis of distant metastases of BC. Pooled sensitivity and specificity of ${ }^{18} \mathrm{~F}-\mathrm{FDG}$ PET/CT were 96\% (95\%CI: 90-98\%) and 95\% (95\%CI: 92-97\%), respectively. Compared with conventional imaging, ${ }^{18} \mathrm{~F}-\mathrm{FDG}$ PET/CT has higher sensitivity for diagnosis of distant metastases in $\mathrm{BC}$ patients.

Similar findings were reported in another meta-analysis by Sun et al. [4]: pooled sensitivity and specificity of ${ }^{18} \mathrm{~F}$-FDG PET or PET/CT were 99\% (95\%CI: $88-100 \%)$ and 95\% (95\%CI: 89-98\%), respectively, confirming the excellent diagnostic performance of ${ }^{18} \mathrm{~F}-\mathrm{FDG}$ PET/CT for distant metastasis staging in $\mathrm{BC}$ patients compared to conventional imaging.

Rong et al. [5] found that the pooled sensitivity and specificity of ${ }^{18} \mathrm{~F}$-FDG PET/CT for detecting bone metastases of $\mathrm{BC}$ were $93 \%(95 \% \mathrm{CI}$ : 82-98\%) and 99\% (95\%CI: 95-100\%), respectively. Compared with bone scintigraphy, 
${ }^{18}$ F-FDG PET/CT has higher sensitivity and accuracy for detection of bone metastases in BC patients.

\subsection{Restaging and Assessment of Response to Neoadjuvant Therapy}

Evangelista et al. [6] performed a meta-analysis on the use of tumour markers in $\mathrm{BC}$ patients as a guide for ${ }^{18} \mathrm{~F}-\mathrm{FDG}$ PET imaging. The metaanalysis provided the following results: pooled sensitivity $87.8 \%$ (95\%CI: 83.8-90.9\%) and pooled specificity $69.3 \%$ (95\%CI: $55.3-80.5 \%$ ), confirming the role of ${ }^{18} \mathrm{~F}-\mathrm{FDG} \mathrm{PET} / \mathrm{CT}$ in detecting metastases in the presence of a progressive increase of serum tumour markers in $\mathrm{BC}$ patients.

Xiao et al. [7] found that the pooled sensitivity and specificity of ${ }^{18} \mathrm{~F}-\mathrm{FDG}$ PET or PET/CT in detecting BC recurrence were $90 \%(95 \% \mathrm{CI}$ : 88-90\%) and 81\% (95\%CI: 78-84), respectively. Therefore, ${ }^{18} \mathrm{~F}$-FDG PET/CT is a valuable imaging method to detect relapse in suspected recurrent $\mathrm{BC}$ patients.

Several meta-analyses evaluated the usefulness of ${ }^{18} \mathrm{~F}-\mathrm{FDG}$ PET/CT in predicting the response to neoadjuvant therapy in $\mathrm{BC}$ patients. According to Wang et al. [8], the pooled sensitivity, specificity, positive predictive value (PPV) and negative predictive value (NPV) of ${ }^{18} \mathrm{~F}-\mathrm{FDG}$ $\mathrm{PET} / \mathrm{CT}$ in this setting were $84 \%(95 \% \mathrm{CI}$ : 78-88\%), 66\% (95\%CI: 62-70\%), 50\% (95\%CI: $44-55 \%$ ) and $91 \%$ (95\%CI: 87-94\%), respectively. For regional lymph nodes, sensitivity and NPV of ${ }^{18} \mathrm{~F}-\mathrm{FDG}$ PET/CT were $92 \%$ (95\% CI: 83-97\%) and 88\% (95\%CI: 76-95\%), respectively. Overall, ${ }^{18} \mathrm{~F}-\mathrm{FDG}$ PET/CT is useful to predict neoadjuvant therapy response in $\mathrm{BC}$ patients, but the relatively low specificity and PPV still call for caution. Cheng et al. [9] found similar results of ${ }^{18} \mathrm{~F}$-FDG PET/CT in this setting reporting a pooled sensitivity and specificity of $84.7 \%$ (95\%CI: 79.3-89.2\%) and 66.1\% (95\%CI: 59.8$72.0 \%)$, respectively, indicating that ${ }^{18} \mathrm{~F}-\mathrm{FDG}$ $\mathrm{PET} / \mathrm{CT}$ has reasonable sensitivity in evaluating response to neoadjuvant chemotherapy in $\mathrm{BC}$, but the specificity is relatively low. Mghanga et al. [10] found that ${ }^{18} \mathrm{~F}-\mathrm{FDG}$ PET has moderately high sensitivity $(80.5 \%$; 95\%CI: $75.9-$ $84.5 \%)$ and specificity (78.8\%; 95\%CI: $74.1-83.0 \%$ ) in early detection of responders from nonresponders, and it can be used for the evaluation of response to neoadjuvant chemotherapy in BC patients. Another meta-analysis [11] reported that the pooled sensitivity and specificity of ${ }^{18} \mathrm{~F}$-FDG PET/CT in this setting were 81.9\% (95\% CI: $76.0-86.6 \%$ ) and 79.3\% (95\% CI: $72.1-85.1 \%$ ), respectively, confirming the moderate accuracy of ${ }^{18} \mathrm{~F}-\mathrm{FDG} \mathrm{PET} / \mathrm{CT}$ in predicting neoadjuvant therapy response in $\mathrm{BC}$ patients.

Several meta-analyses compared ${ }^{18} \mathrm{~F}-\mathrm{FDG}$ PET/CT and MRI for evaluation of treatment response to neoadjuvant chemotherapy (NAC) in $\mathrm{BC}$ patients. Liu et al. [12] reported that ${ }^{18} \mathrm{~F}-\mathrm{FDG}$ PET/CT has a higher sensitivity and MRI has a higher specificity in assessing pathological complete response (pCR) after NAC in BC patients. The pooled sensitivity and specificity of ${ }^{18} \mathrm{~F}-\mathrm{FDG}$ PET/CT were $86 \%$ (95\%CI: 76-93\%) and 72\% (95\%CI: 49-87\%), respectively. Therefore, the combined use of these two imaging modalities may have great potential to improve the diagnostic performance in assessing pCR after NAC. Another meta-analysis [13] indicates that the timing of imaging for NAC-response assessment exerts a major influence on the estimates of diagnostic accuracy: ${ }^{18} \mathrm{~F}-\mathrm{FDG}$ PET/CT outperformed MRI in intra-NAC assessment, whereas the overall performance of MRI was higher after completion of NAC, before surgery. The pooled estimates of sensitivity and specificity were $71 \%$ and $77 \%$ for ${ }^{18} \mathrm{~F}-$ FDG PET/CT and $88 \%$ and $55 \%$ for MRI, respectively. Chen et al. [14] found that the diagnostic performance of MRI is similar to that of ${ }^{18} \mathrm{~F}-$ FDG PET/CT for the assessment of BC response to NAC. For ${ }^{18} \mathrm{~F}-\mathrm{FDG}$ PET/CT, the pooled sensitivity was $87 \%$ (95\%CI: $71-95 \%)$ and pooled specificity was $85 \%$ (95\%CI: 70-93\%). For MRI, the pooled sensitivity was $79 \%$ (95\%CI: $68-87 \%$ ) and the pooled specificity was $82 \%(95 \% \mathrm{CI}$ : $72-89 \%)$. However, ${ }^{18} \mathrm{~F}-\mathrm{FDG}$ PET/CT is more sensitive than conventional contrast-enhanced 
MRI and more specific if the second imaging scan is performed before three cycles of NAC. Lastly, Li et al. [15] found that MRI had a higher sensitivity and ${ }^{18} \mathrm{~F}-\mathrm{FDG}$ PET/CT had a higher specificity in predicting the pathologic response after NAC in patients with $\mathrm{BC}$, with similar accuracy among the two methods. The pooled sensitivity and specificity of MRI were $88 \%$ (95\% CI: 78-94\%), and 69\% (95\%CI: 51-83\%), respectively. The corresponding values for ${ }^{18} \mathrm{~F}-\mathrm{FDG}$ PET/CT were $77 \%$ (95\%CI: 58-90\%) and 78\% (95\%CI: 63-88\%), respectively.

\subsection{Prognostic Value}

Diao et al. [16] evaluated the prognostic value of maximum standardized uptake values (SUVmax) measured in the primary lesion and axillary lymph nodes (ALN) by pretreatment ${ }^{18} \mathrm{~F}$-FDG PET or PET/CT in patients with BC. For eventfree survival (EFS), patients with higher SUVmax in primary tumour and ALN showed a poorer prognosis with pooled hazard ratio (HR) of 1.96 (95\%CI: 1.40-2.73) and 1.89 (95\%CI: 0.70 5.07), respectively. In analysing invasive ductal carcinoma (IDC) patients, the pooled HR was 1.91 (95\%CI: 1.40-2.64). For overall survival (OS), the pooled HR of SUVmax in primary lesion and ALN were 0.64 (95\%CI: $0.23-1.84$ ) and 1.09 (95\%CI 0.07-16.53), respectively. Therefore, patients with BC and higher SUVmax in primary lesion or ALN may experience a higher risk for recurrence or a poor progression.

\subsection{Incidental ${ }^{18}$ F-FDG Uptake}

A meta-analysis calculated the prevalence and clinical significance of breast incidental ${ }^{18} \mathrm{~F}-\mathrm{FDG}$ uptake (BIU) detected by PET or PET/CT in patients performing PET for other reasons than $\mathrm{BC}$ evaluation [17]. The pooled prevalence of BIU on all PET scans was $0.4 \%$ (95\%CI: $0.23-$ $0.61 \%$ ), the pooled prevalence on PET scans on female patients only was $0.82 \%$ (95\% CI: $0.51-$ $1.2 \%$ ), the pooled risk of malignancy of BIU when further evaluated was $48 \%$ (95\%CI: $38-58 \%$ ) and the pooled risk of malignancy of BIU with histological examination was $60 \%$ (95\%CI: 53-66\%). Despite being uncommon, the identification of BIU frequently signals the presence of an unsuspected subclinical lesion and the risk of malignancy is very high.

\section{6 ${ }^{18}$ F-FDG Positron Emission Mammography}

The diagnostic performance of dedicated ${ }^{18} \mathrm{~F}$ FDG positron emission mammography (PEM) in evaluating suspicious $\mathrm{BC}$ has been investigated by a meta-analytic study [18]: pooled sensitivity and specificity of ${ }^{18} \mathrm{~F}-\mathrm{FDG}$ PEM in women with suspected breast malignancy were $85 \%$ (95\%CI: $83-88 \%$ ) and $79 \%$ (95\%CI: $74-83 \%$ ), respectively, on a per-lesion-based analysis. The detection of additional breast lesions and extensive intraductal involvement is improved by PEM, with comparable accuracy over that of MRI in the depiction of invasive $\mathrm{BC}$.

\subsection{PET/MRI}

Lin et al. [19] performed a meta-analysis to assess the staging/restaging performance of hybrid ${ }^{18} \mathrm{~F}-\mathrm{FDG}$ PET/MRI in BC patients. The pooled sensitivity and specificity of ${ }^{18} \mathrm{~F}-\mathrm{FDG}$ PET/MRI for staging/restaging BC were $98 \%$ (95\%CI: 95-99\%) and 87\% (95\%CI: 76-95\%), respectively, on a per-patient analysis and $91 \%$ (95\%CI: 88-94\%) and 95\% (95\%CI: 92-97\%), respectively, on a per-lesion analysis. Overall, ${ }^{18} \mathrm{~F}-\mathrm{FDG}$ PET/MRI has excellent diagnostic performance in staging/restaging $\mathrm{BC}$ patients.

\subsection{Other PET Tracers Beyond ${ }^{18}$ F-FDG}

Evangelista et al. [20] assessed the role of ${ }^{18}$ F-fluoroestradiol $\left({ }^{18} \mathrm{~F}\right.$-FES) PET in patients with BC. A pooled sensitivity of $82 \%$ (95\% CI: 
$74-88 \%$ ) and a pooled specificity of $95 \%$ (95\%CI: 86-99\%) for the evaluation of oestrogen receptor status in $\mathrm{BC}$ by ${ }^{18} \mathrm{~F}-\mathrm{FES}$ PET were found, demonstrating a good accuracy of this method in this setting. Conversely, the pooled sensitivity and specificity of ${ }^{18} \mathrm{~F}$-FES PET in predicting the response to hormonal therapy in patients with locally advanced or metastatic BC were unsatisfactory.

Deng et al. [21] evaluated the diagnostic performance of ${ }^{18} \mathrm{~F}$-fluorothymidine $\left({ }^{18} \mathrm{~F}\right.$-FLT $)$ $\mathrm{PET}$ and PET/CT for evaluating the response to chemotherapy in patients with $\mathrm{BC}$. The pooled sensitivity and specificity of ${ }^{18} \mathrm{~F}$-FLT PET in this setting were $77.3 \%$ (95\%CI: 59.4$90 \%$ ) and $68.5 \%$ (95\% CI: 47.9-84.9\%), respectively, with a moderate diagnostic accuracy.

\section{References}

1. Zhang XH, Xiao C. Diagnostic value of nineteen different imaging methods for patients with breast cancer: a network meta-analysis. Cell Physiol Biochem. 2018;46(5):2041-55.

2. Liang X, Yu J, Wen B, Xie J, Cai Q, Yang Q. MRI and FDG-PET/CT based assessment of axillary lymph node metastasis in early breast cancer: a metaanalysis. Clin Radiol. 2017;72(4):295-301.

3. Hong S, Li J, Wang S. 18FDG PET-CT for diagnosis of distant metastases in breast cancer patients. A meta-analysis. Surg Oncol. 2013;22(2):139-43.

4. Sun Z, Yi YL, Liu Y, Xiong JP, He CZ. Comparison of whole-body PET/PET-CT and conventional imaging procedures for distant metastasis staging in patients with breast cancer: a meta-analysis. Eur J Gynaecol Oncol. 2015;36(6):672-6.

5. Rong J, Wang S, Ding Q, Yun M, Zheng Z, Ye S. Comparison of 18 FDG PET-CT and bone scintigraphy for detection of bone metastases in breast cancer patients. A meta-analysis. Surg Oncol. 2013;22(2):86-91.

6. Evangelista L, Cervino AR, Ghiotto C, Al-Nahhas A, Rubello D, Muzzio PC. Tumor marker-guided PET in breast cancer patients-a recipe for a perfect wedding: a systematic literature review and meta-analysis. Clin Nucl Med. 2012;37(5):467-74.

7. Xiao Y, Wang L, Jiang X, She W, He L, Hu G. Diagnostic efficacy of 18F-FDG-PET or PET/CT in breast cancer with suspected recurrence: a systematic review and meta-analysis. Nucl Med Commun. 2016;37(11):1180-8.
8. Wang Y, Zhang C, Liu J, Huang G. Is 18F-FDG PET accurate to predict neoadjuvant therapy response in breast cancer? A meta-analysis. Breast Cancer Res Treat. 2012;131(2):357-69.

9. Cheng X, Li Y, Liu B, Xu Z, Bao L, Wang J. 18FFDG PET/CT and PET for evaluation of pathological response to neoadjuvant chemotherapy in breast cancer: a meta-analysis. Acta Radiol. 2012;53(6):615-27.

10. Mghanga FP, Lan X, Bakari KH, Li C, Zhang Y. Fluorine-18 fluorodeoxyglucose positron emission tomography-computed tomography in monitoring the response of breast cancer to neoadjuvant chemotherapy: a meta-analysis. Clin Breast Cancer. 2013;13(4):271-9.

11. Tian F, Shen G, Deng Y, Diao W, Jia Z. The accuracy of $18 \mathrm{~F}-\mathrm{FDG} \mathrm{PET} / \mathrm{CT}$ in predicting the pathological response to neoadjuvant chemotherapy in patients with breast cancer: a meta-analysis and systematic review. Eur Radiol. 2017;27(11):4786-96.

12. Liu Q, Wang C, Li P, Liu J, Huang G, Song S. The role of (18)F-FDG PET/CT and MRI in assessing pathological complete response to neoadjuvant chemotherapy in patients with breast cancer: a systematic review and meta-analysis. Biomed Res Int. 2016;2016:3746232.

13. Sheikhbahaei S, Trahan TJ, Xiao J, Taghipour M, Mena E, Connolly RM, et al. FDG-PET/CT and MRI for evaluation of pathologic response to neoadjuvant chemotherapy in patients with breast cancer: a metaanalysis of diagnostic accuracy studies. Oncologist. 2016;21(8):931-9.

14. Chen L, Yang Q, Bao J, Liu D, Huang X, Wang J. Direct comparison of PET/CT and MRI to predict the pathological response to neoadjuvant chemotherapy in breast cancer: a meta-analysis. Sci Rep. 2017;7(1):8479.

15. Li H, Yao L, Jin P, Hu L, Li X, Guo T, et al. MRI and PET/CT for evaluation of the pathological response to neoadjuvant chemotherapy in breast cancer: a systematic review and meta-analysis. Breast. 2018;40:106-15.

16. Diao W, Tian F, Jia Z. The prognostic value of SUVmax measuring on primary lesion and ALN by 18F-FDG PET or PET/CT in patients with breast cancer. Eur J Radiol. 2018;105:1-7.

17. Bertagna F, Treglia G, Orlando E, Dognini L, Giovanella L, Sadeghi R, et al. Prevalence and clinical significance of incidental F18-FDG breast uptake: a systematic review and meta-analysis. Jpn J Radiol. 2014;32(2):59-68.

18. Caldarella C, Treglia G, Giordano A. Diagnostic performance of dedicated positron emission mammography using fluorine-18-fluorodeoxyglucose in women with suspicious breast lesions: a meta-analysis. Clin Breast Cancer. 2014;14(4):241-8.

19. Lin CY, Lin CL, Kao CH. Staging/restaging performance of F18-fluorodeoxyglucose positron emission 
tomography/magnetic resonance imaging in breast cancer: a review and meta-analysis. Eur J Radiol. 2018;107:158-65.

20. Evangelista L, Guarneri V, Conte PF. 18F-Fluoroestradiol positron emission tomography in breast cancer patients: systematic review of the literature \& meta-analysis. Curr Radiopharm. 2016;9(3):244-57.

21. Deng SM, Zhang W, Zhang B, Wu YW. Assessment of tumor response to chemotherapy in patients with breast cancer using (18)F-FLT: a meta-analysis. Chin J Cancer Res. 2014;26(5):517-24.

Open Access This chapter is licensed under the terms of the Creative Commons Attribution 4.0 International License (http://creativecommons.org/licenses/by/4.0/), which permits use, sharing, adaptation, distribution and reproduction in any medium or format, as long as you give appropriate credit to the original author(s) and the source, provide a link to the Creative Commons license and indicate if changes were made.

The images or other third party material in this chapter are included in the chapter's Creative Commons license, unless indicated otherwise in a credit line to the material. If material is not included in the chapter's Creative Commons license and your intended use is not permitted by statutory regulation or exceeds the permitted use, you will need to obtain permission directly from the copyright holder. 